\title{
A New Approach to Detect Epileptic Seizures in Electroencephalograms Using Teager Energy
}

\author{
Chandrakar Kamath \\ Electronics and Communication Department, Manipal Institute of Technology, Manipal 576104, India \\ Correspondence should be addressed to Chandrakar Kamath; chandrakar.kamath@gmail.com
}

Received 6 April 2013; Accepted 4 May 2013

Academic Editors: L. Faes and R. Grebe

Copyright ( 2013 Chandrakar Kamath. This is an open access article distributed under the Creative Commons Attribution License, which permits unrestricted use, distribution, and reproduction in any medium, provided the original work is properly cited.

\begin{abstract}
A Teager energy (TE) based approach to discriminate electroencephalogram signals corresponding to nonseizure (eyes open, eyes closed, or interictal) and seizure (ictal) intervals is proposed. Though a good number of contributions have been made for seizure detection, the challenges of unbalanced data (nonseizure and seizure events) and system computational efficiency still remain a challenge. It is reported in the literature that the seizures are characterized by abnormal sudden discharges in the brain which get manifested in the EEG recordings by frequency changes and increased amplitudes. Teager energy (TE) is capable of tracking such rapid changes in frequency as well as amplitude in the time domain. An important finding of this study is that the mean TE quantifier is largely independent of the window length and exhibits relative consistency when used as a relative measure for comparison. We compared the diagnostic capability of TE quantifier with those of Higuchi's fractal dimension and sample entropy in discriminating nonseizure and seizure states in the EEGs and found that TE outperforms the other two nonlinear quantifiers. The result shows that the application of this method compares favorably with conventional classification methods in terms of performance and is well suited for real-time automatic epileptic seizure detection.
\end{abstract}

\section{Introduction}

Electroencephalography (EEG) is an important noninvasive clinical tool for monitoring, diagnosing, and managing neurological disorders related to epilepsy. Epilepsy is the most common serious neurological disorder after stroke, and approximately $1 \%$ of the world's population suffers from this disorder [1]. For most of the patients seizures occur suddenly and unexpectedly without any prior external precipitants. The unforeseen nature of these seizures makes the daily life miserable with temporary impairments of perception, speech, memory, motor control, and/or consciousness and sometimes may lead to enhanced risk of injury and/or death. A person is diagnosed as epileptic, only if there is a recurrent tendency to have seizures. Epilepsy can be controlled but not cured with antiepileptic medication. The epileptic brain can be considered to function in one of the two states: interictal state with occasional transient waveforms, as isolated spikes, sharp waves, or spike-wave complexes, and ictal state with continuous discharge of polymorphic waveforms of varying amplitude and frequency, spike and sharp wave complexes, and rhythmic hypersynchrony [2]. The EEG during seizure is significantly different from that of the interictal state and that of a normal subject. The conventional methods rely on experts who visually inspect the entire lengthy EEG signals recorded over several days, which is tedious, time-consuming and costs higher. Therefore, many automated epileptic detection systems have been developed using different approaches in the recent years [3]. Such automated systems reduce the time taken to review offline the long-term EEG recordings significantly and facilitate the neurologist to diagnose and treat more patients in a given time. If, in addition, an online seizure detection system is made available, then the system can provide a warning signal/alarm to healthcare professionals to pay immediate attention when the seizure occurs. This implies that the selected feature must be such that, besides accuracy in seizure detection, the processing time must be very short.

Automated epileptic seizure analysis refers collectively to methods for (i) epileptic seizure detection, (ii) epileptic 
seizure prediction, and (iii) automatic focus channel identification. These methods are primarily applied on EEG signals $[4,5]$. In this study, however, the focus is on epileptic seizure detection. The first remarkable contribution to automatic detection of epileptic seizures/patients was made by Gotman [6], in which EEG signal was decomposed into elementary waves followed by the detection of paroxysmal bursts of rhythmic activity having a frequency between 3 and $20 \mathrm{~Hz}$. The amplitude of these waves relative to the background and their duration and rhythmicity served as measures to distinguish seizures. Murro et al. developed an automated seizure detection system based on the discriminant analysis of the EEG signal recorded from the intracranial electrodes [7]. Nearest-neighbor classifier, with the features from both the time and frequency domains as input, has been tried to detect the onset of the epileptic seizures [8]. Radhakrishnan and Gangadhar used approximate entropy and Lempel-Ziv complexity (LZC) as measures to quantify the regularity in the various epochs of epileptic seizure time series data [9]. Hu et al. compared LZC with correlation entropy in the context of epileptic seizures detection from EEG data and discussed the advantages of the normalized LZC over the correlation entropy [10]. Analysis of EEG using chaotic nonlinear dynamics, such as Lyapunov exponents (LEs), has been proposed to study seizure discharges $[11,12]$. Based on the evolution of accumulated energy using wavelet analysis Gigola et al. predicted the onset of epileptic seizure from intracranial EEG recordings [13]. Other researchers have employed different entropies (wavelet entropy, permutation entropy, and approximate entropy) [14-17] and complexity measures (Kolmogorov entropy, Hurst exponent, correlation dimension, scale dependent LE, and multiscale complexity measure) for characterizing EEG and to indicate epileptic seizures $[18,19]$.

Selecting appropriate features that best represent the characteristics of EEG signals is important in seizure detection and variety of features have been investigated as mentioned above [6-19]. However, the challenges of unbalanced data (nonseizure and seizure states) and system computational efficiency still remain a challenge. The rare occurrence of an epileptic seizure over hours of EEG recordings makes training and testing of the model/classifier difficult due to the bias towards the nonseizure class. System computational efficiency becomes a challenge when moving to real-time applications. To circumvent these problems, in this work, we employ mean nonlinear energy estimator derived from a noise robust version of the Teager energy operator (TEO) for the classification of EEG signals (see Section 2.2 below for details) [20-22]. The rationale of adopting nonlinear Teager energy (TE) as a quantifier in this study is based on the following reasons. (1) Seizures are abnormal sudden discharges in the brain which are represented in the EEG recordings by frequency changes and increased amplitude. The ability to accurately capture these changes is the key to detection of nonseizure and seizure states. TE is a feature very sensitive to variations of signal amplitude and frequency. (2) TE feature requires only four samples for its computation at any given instant and hence is computationally very efficient. (3) It has been found that physiological changes in the
EEG, usually associated with the epilepsy, include spikes, sharp waves, subclinical seizures, and increased energy in the signal [23]. This abnormal neuronal activity can last from several seconds to minutes. TE is characterized by a time resolution that can capture such rapid changes in the modulation energy of the signal (see Section 2.2 below for details) [24]. We show that the mean TE quantifier can be used for short-term as well as long-term analysis. (4) EEG signals are known to be nonstationary in nature $[23,25]$. We hypothesize that epochs of normal, interictal epileptic and seizure belong to different nonlinear physiological processes. TEO is a nonlinear operator which can be used for the estimation of energy of a nonstationary signal and, hence TE is well suited for discriminating these episodes.

TE based features have been widely used in nonlinear speech analysis and processing [26-28]. TEO has also been used in the detection of congestive heart failure and classification of electrocardiograms (ECGs) $[29,30]$. Several successful attempts at seizure detection using TE features have been reported [31-35]. Zaveri et al. employed conventional measure of energy and Teager's algorithm to estimate the energy of the electrocorticograms (ECOG) recorded in temporal lobe epilepsy [31]. The study evaluated the propagation of seizure after the onset. However, in comparison with other tools such as ECOG, EEG is a clean and safe technique for monitoring the brain activity. Esteller found 31 distinct features, comprising fractional dimension, wavelet packet energy, and Teager energy, as suitable for seizure detection [32]. Curve length, mean energy, and Teager energy were used as input to a support vector machine by Gardner et al. for detecting seizures in humans [33]. Agarwal and Gotman used a nonlinear energy operator to characterize EEG signal adaptive segmentation of the signal into piecewise stationary segments [34]. Changes in the amplitude or frequency of the EEG signal facilitated the demarcation and provided indicators as to the spectral content of the signal. D'Alessandro et al. employed TEO output as one of the hybrid features in the prediction of epileptic seizures [35]. Their method provides a structure based upon multiquantitative features and multielectrode sites that can be used to optimize seizure prediction for a particular group of features. In all the methods [31-35], a modern classifier was employed to first train and then classify the EEG to belong to either interictal or ictal class. Moreover, all of them used a multifeature vector which increases the computational complexity and time. Yadav et al. have used frequency weighted energy (FWE), derived from the general form of the Teager operator, in the detection of seizures in stereoencephalograms [36]. They found the combination $l=1, m=2, p=0$, and $q=4$ to be a better choice in the Teager equation. Single channel intracerebral EEG records from five patients were used in the study. They used a 2-second moving window shifted in steps of 0.25 seconds to compute the mean FEW. The method was gradient based, and any activity that crossed the minimum duration and met some specific criteria was deemed to be seizure activity. The prime advantage of their method is that it is threshold independent. The conventional nonlinear quantifiers have some drawbacks. Firstly, most of them are window-length dependent and require a large number of 
data samples to arrive at statistically reliable and reproducible results. Secondly, they assume the signals to be stationary and noise free. Unfortunately it is difficult for physiological signals to meet these criteria. Thirdly, most of them depend on parameters to compute them. In this study, we employ the mean TE quantifier, which we show: (1) to be largely independent of window length, (2) to be suitable for shortterm as well as long-term analysis, (3) not to require any parameters for computation, and (4) to demonstrate relative consistency when used as a relative measure for comparison. This property enables the separation of the different EEG states almost independent of window length. A threshold procedure is used to make a final decision. We demonstrate the power of mean TE quantifier in discriminating eyes open and eyes closed episodes (from normal subjects), interictal, and ictal episodes (from epileptic patients) in EEG signal, for different window lengths (varying from about 1 second to 23 seconds), by comparing its performance with those of other two nonlinear quantifiers, namely, Higuchi's fractal dimension and sample entropy [37-42]. Such discrimination is important in some applications, such as seizure warning systems, closed loop seizure control systems, or delivering abortive responses/monitoring patients using implantable therapeutic devices [43]. We also compare the performance of our approach with those of other researchers who had used the same database by Andrzejak et al. [44]. The presented method is simple, computationally very efficient and, hence is well suited for real -time implementation.

\section{Methods and Materials}

2.1. EEG Records. The EEG data used for this work is from University of Bonn EEG database which is available in public domain [44]. The choice of this database is based on the rationale that many seizure detection methods have employed this database, and it becomes easy to compare the end results. The database consists of five sets (designated $\mathrm{Z}, \mathrm{O}$, $\mathrm{N}, \mathrm{F}$, and S) each containing 100 single channel EEG segments of 23.6 second duration. These segments have been picked from continuous multichannel EEG recordings after removal of any artifacts, like, muscle activity, or eye movements, making sure that they fulfilled stationarity requirements. Sets $\mathrm{Z}$ and $\mathrm{O}$ contain segments taken from surface EEG recordings acquired from five healthy volunteers using a standard 10-20 electrodes placement scheme. The subjects were awake and relaxed with their eyes open for set $\mathrm{Z}$ and eyes closed for set $\mathrm{O}$, respectively. The segments for sets $\mathrm{N}, \mathrm{F}$, and $\mathrm{S}$ were acquired from five epileptic patients undergoing presurgical diagnosis. The type of epilepsy identified was temporal lobe epilepsy with the epileptogenic focus as the hippocampal formation. These recordings were taken from intracranial electrodes as they offer the most precise access to the emergence of seizures. Sets $\mathrm{N}$ and $\mathrm{F}$ contained only activity measured during seizure-free intervals (interictal epileptiform activity), with segments in set $\mathrm{N}$ recorded from hippocampal formation of the opposite hemisphere of the brain and those in set $\mathrm{F}$ recorded within epileptogenic zone. On the other hand, set $S$ contained only seizure activity (ictal intervals), with all segments recorded from sites exhibiting ictal activity. The patients had attained complete seizure control after resection of one of the hippocampal formations which was confirmed to be the epileptogenic zone. All the EEG signals were recorded using the same 128-channel amplifier system using an average common reference. The data were digitized at 173.6 samples per sec with 12 bit resolution. The bandpass filter setting was at $0.53-40 \mathrm{~Hz}$ (12 dB/octave). Each single channel EEG segment has 4096 samples. Empirically we found that an analysis window of 3000 samples (17.3 seconds) leads to nearoptimum results (see Section 3 for details). Hence, 3000sample sliding window with 2700 samples (90\%) overlap is used with every EEG segment from each dataset. This procedure leads to, in effect, 2000 analysis EEG segments in each of the five datasets ( $\mathrm{Z}, \mathrm{O}, \mathrm{N}, \mathrm{F}$, and $\mathrm{S})$. This of course increases statistical reliability and reproducibility of this study.

In this work, we employ four different classification problems and compare the performance of our approach with those of two powerful and popular nonlinear quantifiers, Higuchi's fractal dimension and sample entropy, and also of other researchers. The first three classifications are as proposed by Guo et al. [45] in the literature. In the first classification problem, two classes are examined, normal and seizure. The normal class includes only set $\mathrm{Z}$ while seizure class includes set $S$. In this classification problem, 4000 analysis EEG segments are included. In the second classification two classes, namely, nonseizure and seizure, are examined. The nonseizure class includes sets $\mathrm{Z}, \mathrm{N}$, and $F$ while seizure class includes set $S$. In this classification problem, the dataset includes 8000 analysis EEG segments. In the third problem, again, two classes, nonseizure and seizure, are examined. Now the nonseizure class includes sets $\mathrm{Z}, \mathrm{O}, \mathrm{N}$, and $\mathrm{F}$ while seizure class includes set $\mathrm{S}$. In this classification problem, 10000 analysis EEG segments are included in the dataset. The fourth classification problem considers data only from epileptic patients. In this case, one is an interictal class which includes $\mathrm{N}$ and $\mathrm{F}$ sets, while the other includes ictal (seizure) set S. This problem uses 6000 analysis EEG segments. These classification problems have been chosen such that they are close to clinical applications.

2.2. Teager Energy Operator (TEO). The rationale of adopting nonlinear Teager energy (TE) as a quantifier in this study has already been discussed under the introduction section. An interesting feature of TEO is that it is characterized by a time resolution that can trace rapid changes in the modulation energy (squared product of amplitude and frequency) of the signal. Although the energy of any two tones at different frequencies, but equal amplitude, is the same, the energy required to generate the two tones is different. The higher the frequency of the tone. The higher the frequency of the tone, more is the energy required for its generation, and TE reflects this energy. Since the operator can track amplitude envelope and instantaneous frequency (as shown below), the algorithm is recognised as "nonlinear energy" [35].

If a signal sample is represented as $x_{n}=A \cos (\Omega n+\Phi)$, where $A$ is the amplitude and $\Phi$ is the initial phase, then $\Omega$ is 
the digital frequency in radians/sample and is given by $\Omega=$ $2 \pi f / f_{s}$, where $f$ is the analog frequency in $\mathrm{Hz}$ and $f_{s}$ is the sampling frequency in $\mathrm{Hz}$. Then as per the TE algorithm, the instantaneous TE, $E_{n}$ at a given instant of time $n$, is given by [24]

$$
\begin{gathered}
E_{n}=x_{n}^{2}-x_{n-1} x_{n+1}=A^{2} \sin ^{2}(\Omega) \\
\mathrm{E}_{n} \approx A^{2} \Omega^{2}
\end{gathered}
$$

for small $\Omega$. With $\Omega<\pi / 4$ or $f / f_{s}<1 / 8$ the relative error in the last approximation is always less than $11 \%$. From (1) it is clear that the instantaneous TE can track modulation energy and identify instantaneous signal amplitude and also corresponding instantaneous frequency. For example, in a normal subject there is a fine balance in the brain between factors that generate electrical activity and factors that restrict it, and there are also systems that limit the spread of the electrical activity. Usually, during a seizure, these limits breakdown, and an abnormal hypersynchronous neuronal activity due to a large number of neurons in the cerebral cortex of the brain occurs. The cerebral activity during an epileptic seizure is completely different from that of the interictal state or that of a normal subject. During interictal state the EEG is normal with occasional transient waveforms and apparently random with higher complexity, while during seizure the EEG tends to become hypersynchronized and cyclical with decreased complexity $[46,47]$. Unlike the usual instantaneous signal energy which is only proportional to squared instantaneous amplitude, TE is proportional to the squared product of both instantaneous amplitude and instantaneous frequency. This new energy measure is therefore capable of responding rapidly to changes in both amplitude and frequency. Consequently disturbances in EEG signal generation and conduction path get reflected in the TEO energy $[29,30]$.

The general form of Teager nonlinear energy operator in the time domain for a discrete time signal $x[n]$ as given by Plotkin and Swamy $[20-22,34]$ is

$$
\Psi_{\mathrm{td}}\{x[n]\}=x[n-l] x[n-m]-x[n-p] x[n-q],
$$

where $l+m=p+q$ and $\Psi_{\text {td }}$ denotes generalized TEO.

They showed that, for $l \neq m$ and $p \neq q, \Psi_{\text {td }}$ is very robust to noise. That is, if the input signal contains additive white noise, then the output of (2) will not contain a component related to input noise. This is attributed to the removal of the square term in (2) while satisfying the conditions $l+m=p+q$, $l \neq m$, and $p \neq q$. In this work, we empirically found that the combination $l=1, m=2, p=0$, and $q=3$ is a suitable choice for noise reduction in EEG signals. It is to be noted that TEO defined by (1) uses only three samples, while the noise robust version defined by (2) uses four samples. The average nonlinear energy in the time domain, $\mathrm{ANE}_{t}$, is defined as [35]

$$
\mathrm{ANE}_{\mathrm{td}}=\log _{10}\left(\frac{\left(\sum_{n} \Psi_{\mathrm{td}}\{x[n]\}\right)}{N}\right), \quad 0 \leq n \leq N-1
$$

where $N$ is the number of samples in $\Psi_{\mathrm{td}} . \mathrm{ANE}_{\mathrm{td}}$, the mean TE quantifier (on logarithmic scale), characterizes changes in nonlinear energy of EEG signal in the time domain. $\mathrm{ANE}_{\mathrm{td}}$ can be thought of as a mean (modulation) energy estimator, in which the instantaneous nonlinear energy is computed using (2). Higher values indicate higher differences in the instantaneous amplitude and/or instantaneous frequency of the signal. Considering that a sliding window is used, we get the moving average of the nonlinear energy of the EEG signal. It is found that the hypersynchronous neuronal activity during seizures considerably increases this nonlinear energy.

2.3. Higuchi's Fractal Dimension (HFD). FD primarily quantifies complexity or self-similarity of a signal. Higuchi proposed a simple, accurate, and fast method to compute the fractal dimension of a signal $[48,49]$. It represents the FD of a curve representing the amplitude of the signal under investigation on a plane as a function of time, which at the same time serves as a measure of complexity and selfsimilarity of the signal. It is to be noted that Higuchi's fractal dimension (HFD) is completely different from the correlation dimension. If HFD is computed using a moving time window, then the resulting HFD characterizes the changes in signal complexity. Lower values of HFD imply decrease in dimensional complexity and vice versa. Abrupt changes in HFD represent nonstationarities in the signal [50].

Let $x(1), x(2), \ldots, x(N)$ represent a one-dimensional discrete time sequence. The Higuchi fractal dimension is based on a measure of length $L(k)$ of the curve that represents the original time series while using a segment of $\mathrm{k}$ samples as a unit [50]. If $L(k)$ scales like

$$
L(k) \approx k^{-D}
$$

where $D$ is called fractal dimension and is a measure of complexity and irregular characteristics of the time series, then for a simple curve $D=1$ and for a curve which fills out the entire plane $D=2$.

From the original time series: $x(1), x(2), \ldots, x(N), k$ number of new time series are constructed according to Higuchi's algorithm. The new time series is given by

$$
\begin{array}{r}
x_{m}{ }^{k}=\{x(m), x(m+k), x(m+2 k), \ldots, \\
\left.x\left(m+\operatorname{int}\left(\frac{(N-m)}{k}\right) * k\right)\right\}, \\
m=1,2, \ldots, k,
\end{array}
$$

where $N$ is the total number of samples, $m$ is the initial time, $k$ is the interval time, and $\operatorname{int}(r)$ is the integer part of a real number $r$. The length $L_{m}(k)$, of each time series $x_{m}{ }^{k}$, is then given by

$$
\begin{aligned}
& L_{m}(k) \\
& \qquad=\left|\frac{(N-1) \sum_{i}\{|x(m+i * k)-x(m+(i-1) * k)|\}}{(k *(M * k))}\right|, \\
& 1 \leq i \leq N-2,
\end{aligned}
$$


where,

$$
M=\operatorname{int}\left(\frac{(N-m)}{k}\right) .
$$

It is to be noted that $L_{m}(k)$ is not a distance in the Euclidean sense. Instead it represents the normalized sum of absolute values of difference in ordinate pairs of points distant $k$ (with the initial point $m$ ). Then the length of the curve for the time interval $k$ is computed as the average of the $k$ number of $L_{m}(k)$ values, as defined by

$$
L(k)=\frac{\left(\sum_{m} L_{m}^{k}\right)}{k}, \quad 1 \leq m \leq k .
$$

Finally $L(k)$ is plotted against $1 / k$ on $\log$-log scale for $k=$ $1,2, \ldots, k_{\max }$. The slope of the resulting curve that fits the pairs of points $\{\ln (L(k)), \ln (1 / k)\}$ in the least square sense constitutes the fractal dimension of the given time series.

The best value for the maximum number of time series constructed, $k_{\max }$, is to be found by the following procedure. As mentioned above, for each constructed time series the curve length is computed and plotted against the corresponding $1 / k$ value on a log-log scale. The slope of the resulting curve represents the fractal dimension of the given time series. Next the fractal dimensions are plotted for different values of $k_{\max }$. The point at which the fractal dimension plateaus corresponds to the best value of $k_{\max }$ beyond which the $k_{\max }$ value does not yield better results. In this work, we found that value of $k_{\max }=2$ was sufficient. The HFD is calculated over an analysis window of 3000 samples of the EEG time series (with 90\% overlap) from each dataset.

2.4. Sample Entropy (SampEn). The prime advantage of sample entropy is that "it is largely independent of record length and shows relative consistency where approximate entropy does not" [41]. The sample entropy represents the negative natural logarithm of the conditional probability that two sequences similar for $m$ points remain similar at the next point [42]. Given a time series with $N$ data points, $x_{1}, x_{2}, \ldots, x_{N}$, and to compute SampEn $m$-dimensional vector sequences, $y_{i}(m)=\left\{x_{i}, x_{i+1}, \ldots, x_{i+m-1}\right\}$, where $1 \leq i \leq$ $N-m+1$. Then

$$
\begin{array}{r}
B_{i}^{m}(r)=\frac{\left(\sum_{j} \theta\left(r-\left\|y_{j}(m)-y_{i}(m)\right\| \infty\right)\right)}{(N-m-1)} \\
\text { for } j \neq i, 1 \leq j \leq N-m,
\end{array}
$$

where $\theta$ is the Heaviside function and $\|\cdot\|_{\infty}$ is the maximum norm defined by $\left\|y_{j}(m)-y_{i}(m)\right\|_{\infty}=\max _{0 \leq k \leq m-1} \mid x_{j+k}-$ $x_{i+k} \mid$. The sum in the above equation represents the number of vectors $y_{j}(m)$ that are within a circular distance $r$ from $y_{i}(m)$ in the reconstructed phase space. However, the cases of selfmatches indicated by $j=i$ are avoided from the count. This brings down the bias in the estimation of SampEn [41]. In the next step the density is computed as

$$
B^{m}(r)=\frac{\left(\sum_{i} B_{i}^{m}(r)\right)}{(N-m)} \quad \text { for } 1 \leq i \leq N-m .
$$

Computations similar to the above are then performed on a $(m+1)$-dimensional reconstructed space to arrive at the following equations:

$$
\begin{gathered}
A_{i}^{m}(r)=\frac{\left(\sum_{j} \theta\left(r-\left\|y_{j}(m+1)-y_{i}(m+1)\right\|_{\infty}\right)\right)}{(N-m-1)} \\
\text { for } j \neq i, 1 \leq j \leq N-m, \\
A^{m}(r)=\frac{\left(\sum_{i} A_{i}^{m}(r)\right)}{(N-m)} \quad \text { for } 1 \leq i \leq N-m .
\end{gathered}
$$

We thus have

$$
\begin{aligned}
& B(r)=\frac{(N-m-1)(N-m) B^{m}(r)}{2}, \\
& A(r)=\frac{(N-m-1)(N-m) A^{m}(r)}{2} .
\end{aligned}
$$

$B(r)$ and $A(r)$, respectively, represent the total number of template matches in an $m$-dimensional and $(m+1)$ dimensional phase space within a tolerance $r$.

The sample entropy being defined as the negative of natural logarithm of the conditional probability that a dataset of length $N$, having repeated itself for $m$ samples within a specified tolerance $r$, will also repeat itself for $m+1$ samples without allowing self-matches; we compute the same as

$$
\operatorname{SampEn}(m, r, N)=-\log \left(\frac{A(r)}{B(r)}\right) .
$$

For this work, we empirically found that $m=3, d=0.1 * \mathrm{SD}$ yield good results. The SampEn is calculated over an analysis window of 3000 samples of the EEG time series (with $90 \%$ overlap) from each dataset. Higher values indicate increased irregularity in the signal and vice versa.

2.5. Statistical and Receiver Operating Characteristic (ROC) Analyses. First we evaluate the statistical significance of the three nonlinear analysis methods, TE, HFD, and SampEn, using Kruskal-Wallis test on the results of each classification problem. Parameters are regarded as statistically significant if $P<0.01$. If significant differences between classes are found, then the ability of the nonlinear analysis method to discriminate one class from the other is evaluated using receiver operating characteristic (ROC) plots in terms of the area under ROC curve (AUC). ROC plots are used to gauge the predictive ability of a classifier over a wide range of values [51]. A threshold value is applied such that a feature value below this threshold will be assigned category1 while a feature value above the threshold will be assigned category-2. ROC curves are obtained by plotting sensitivity values (which represent the proportion of the subjects with positive diagnosis for category-1) along the $y$-axis against the corresponding (1-specificity) values (which represent the proportion of the correctly identified category-2) for all the available cutoff points along the $x$-axis. Accuracy is a related parameter that quantifies the total number of subjects (both category-1 and category-2) precisely classified. The AUC 


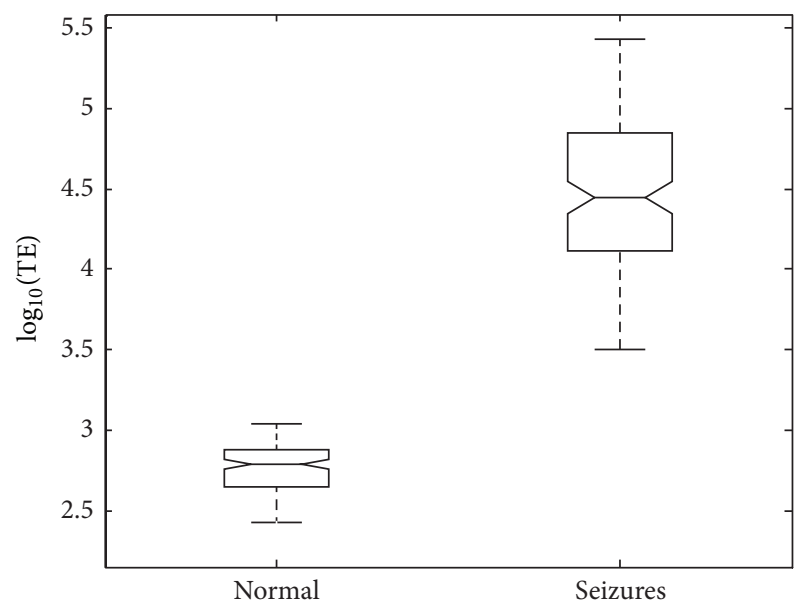

(a)

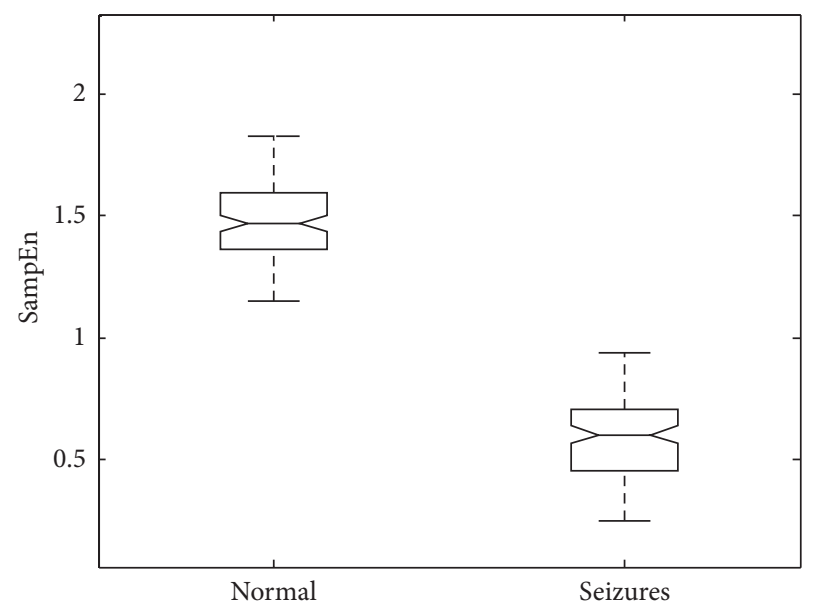

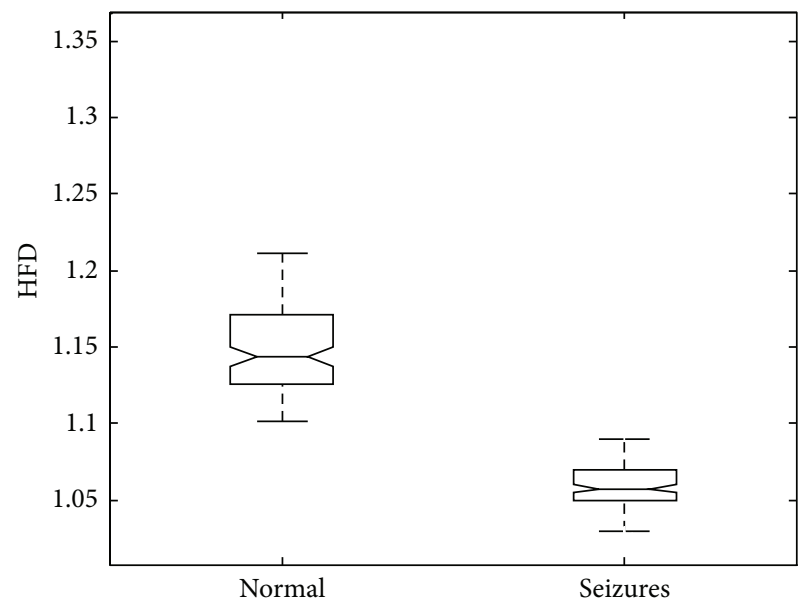

(b)

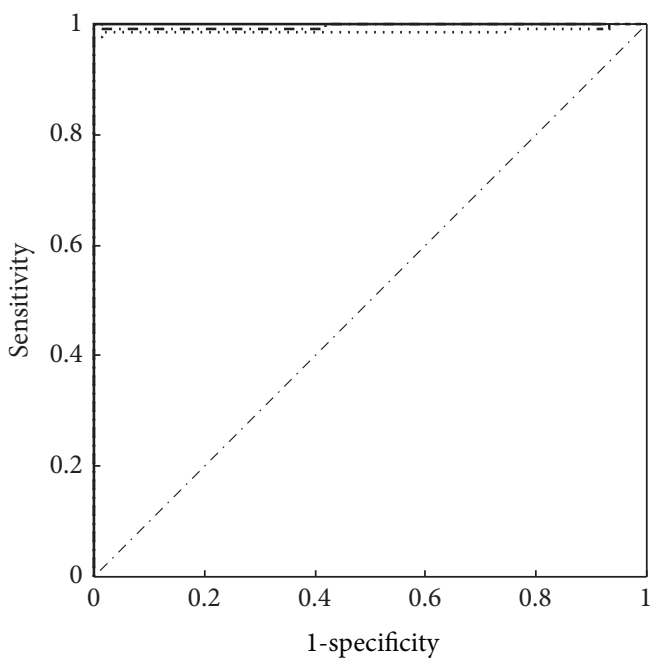

$\begin{array}{ll}- & \log _{10}(\mathrm{TE}) \\ \ldots . . & \text { HFD } \\ \ldots & \text { SampEn }\end{array}$

(c)

(d)

Figure 1: For classification problem-1 (a) the distribution of $\log _{10}$ (TE) values using box-whiskers plots (without outliers) for normal and seizure groups. (b) The distribution of HFD values using box-whiskers plots (without outliers) for normal and seizure groups. (c) The distribution of SampEn values using box-whiskers plots (without outliers) for normal and seizure groups. (d) ROC curve for log ${ }_{10}$ (TE) (thick solid line), HFD (thick dotted line), and SampEn (thick dash-dot line) for discriminating normal and seizure groups. The diagonal line (thin dash-dot line) from 0,0 to 1,1 represents ROC curve that cannot discriminate between normal and seizure groups.

TABle 1: $P$ value and Chi-sq. values of Kruskal-Wallis test for TE, HFD, and SampEn of different classification problems for $W=3000$.

\begin{tabular}{lccccc}
\hline Classification problem & \multicolumn{2}{c}{ TE } & \multicolumn{2}{c}{ HFD } & \multicolumn{2}{c}{ SampEn $(3,0.1 *$ SD, $W)$} \\
& Chi-sq & $P$ & Chi-sq. & $P$ & Chi-sq. \\
\hline 1 & 203.25 & 0 & 193.23 & 0 & 200.76 \\
2 & 303.39 & 0 & 263.83 & 0 & 191.85 \\
3 & 320.76 & 0 & 235.00 & 0 & 229.70 \\
4 & 268.61 & 0 & 223.00 & 0 & 148.60 \\
\hline
\end{tabular}




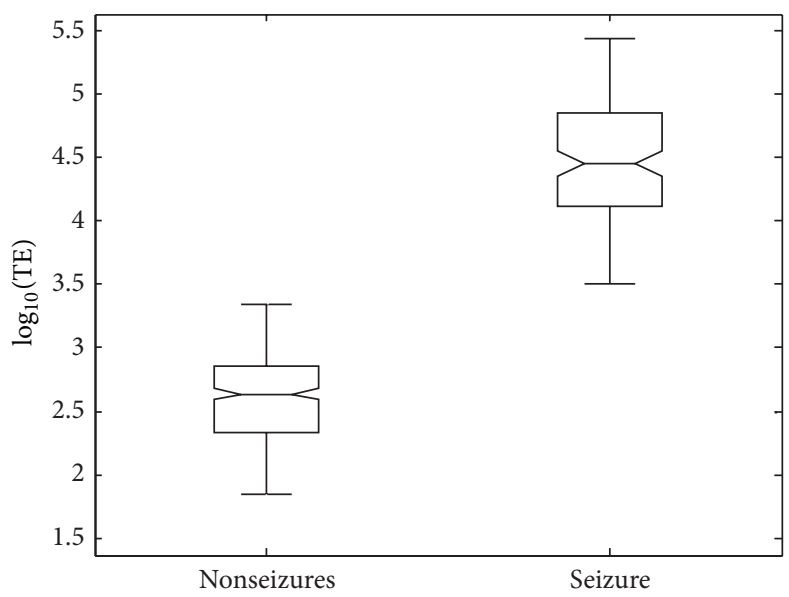

(a)

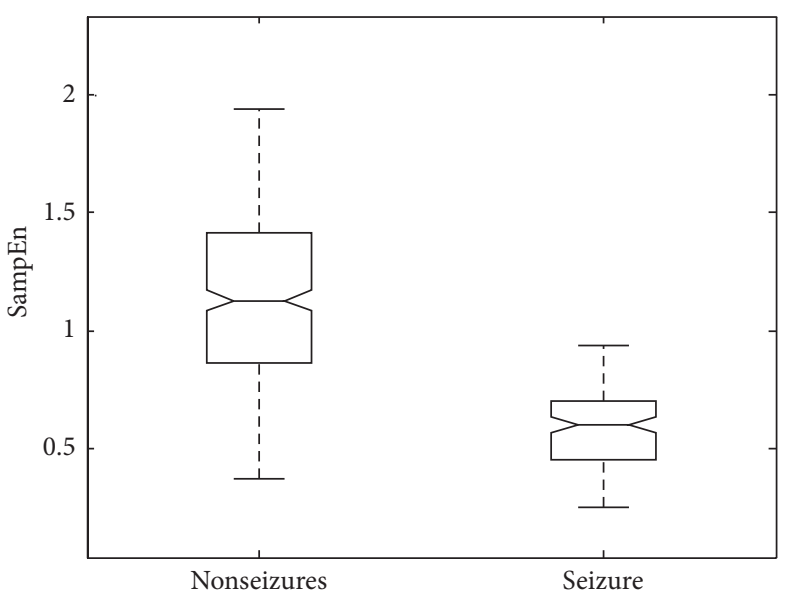

(c)

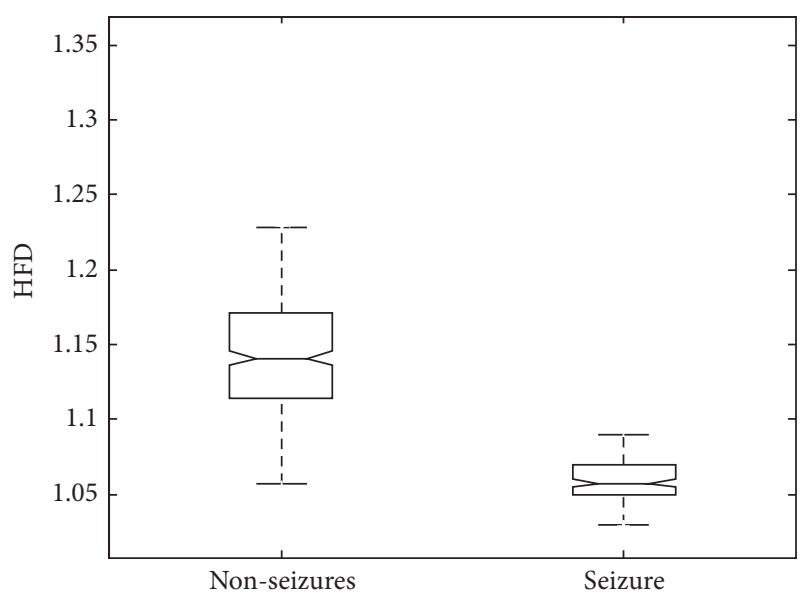

(b)

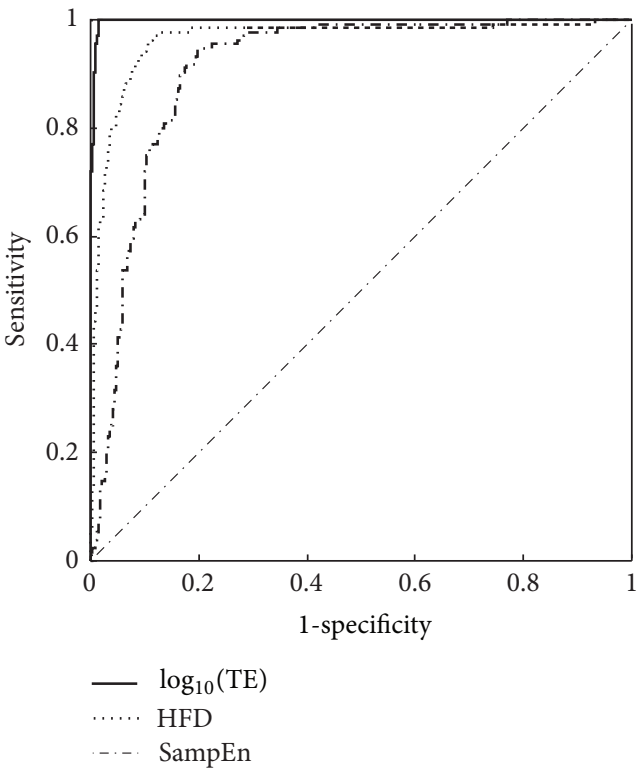

(d)

FIGURE 2: For classification problem-2 (a) the distribution of $\log _{10}$ (TE) values using box-whiskers plots (without outliers) for nonseizure and seizure groups. (b) The distribution of HFD values using box-whiskers plots (without outliers) for nonseizure and seizure groups. (c) The distribution of SampEn values using box-whiskers plots (without outliers) for nonseizure and seizure groups. (d) ROC curve for log $\log _{10}$ (TE) (thick solid line), HFD (thick dotted line), and SampEn (thick dash-dot line) for discriminating nonseizure and seizure groups. The diagonal line (thin dash-dot line) from 0,0 to 1,1 represents ROC curve that cannot discriminate between nonseizure and seizure groups.

TABLE 2: Descriptive results of ROC analysis using TE, HFD, and SampEn for discriminating normal and seizure groups (classification problem-1) for $W=3000$.

\begin{tabular}{lcccccc}
\hline Nonlinear method & AUC & Optimum threshold & Average sensitivity \% & Average specificity \% & Average precision \% & Average accuracy \% \\
\hline TE & 1.0000 & 3.1937 & 100.0 & 100.0 & 100.0 & 100.0 \\
HFD & 0.9875 & 1.1069 & 98.5 & 98.5 & 98.5 & 98.5 \\
SampEn & 0.9969 & 1.0378 & 99.3 & 100.0 & 100.0 & 99.6 \\
\hline
\end{tabular}

TABLE 3: Descriptive results of ROC analysis using TE, HFD, and SampEn for discriminating nonseizure and seizure groups (classification problem-2) for $W=3000$.

\begin{tabular}{lcccccc}
\hline Nonlinear method & AUC & Optimum threshold & Average sensitivity \% & Average specificity \% & Average precision \% & Average accuracy \% \\
\hline TE & 0.9983 & 3.5082 & 99.3 & 98.8 & 96.4 & 98.9 \\
HFD & 0.9647 & 1.0865 & 94.1 & 90.7 & 77.1 & 91.5 \\
SampEn & 0.9123 & 0.7761 & 89.7 & 83.6 & 64.6 & 85.1 \\
\hline
\end{tabular}




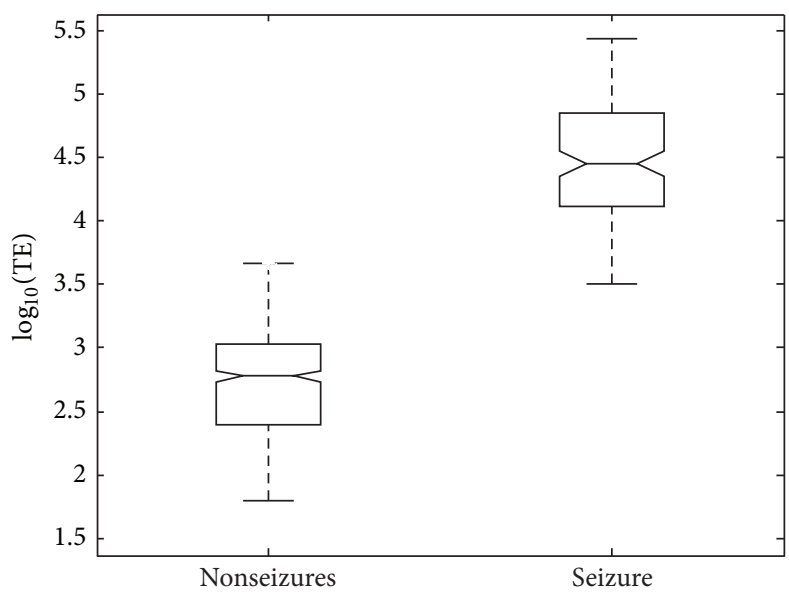

(a)

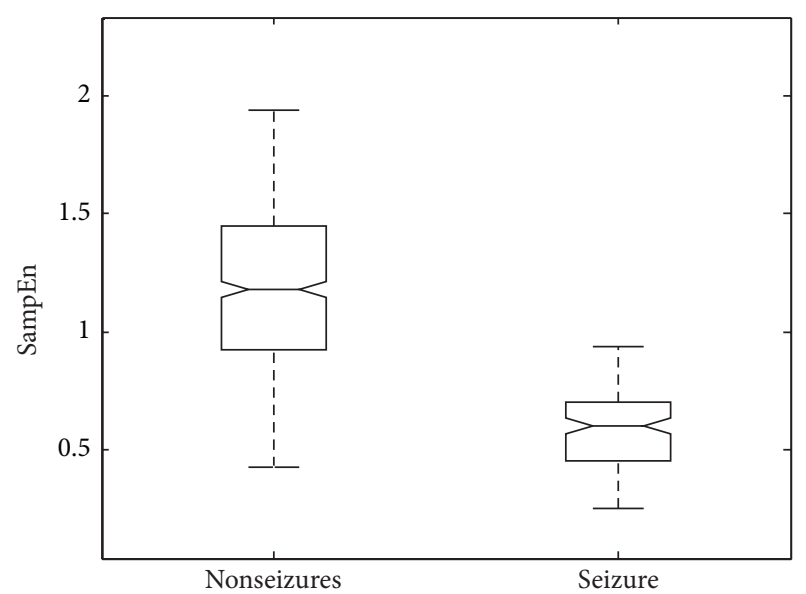

(c)

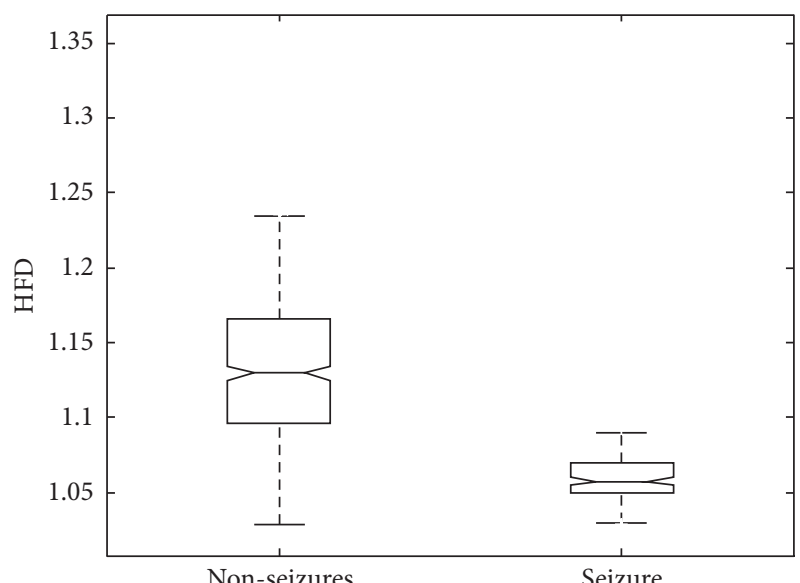

(b)

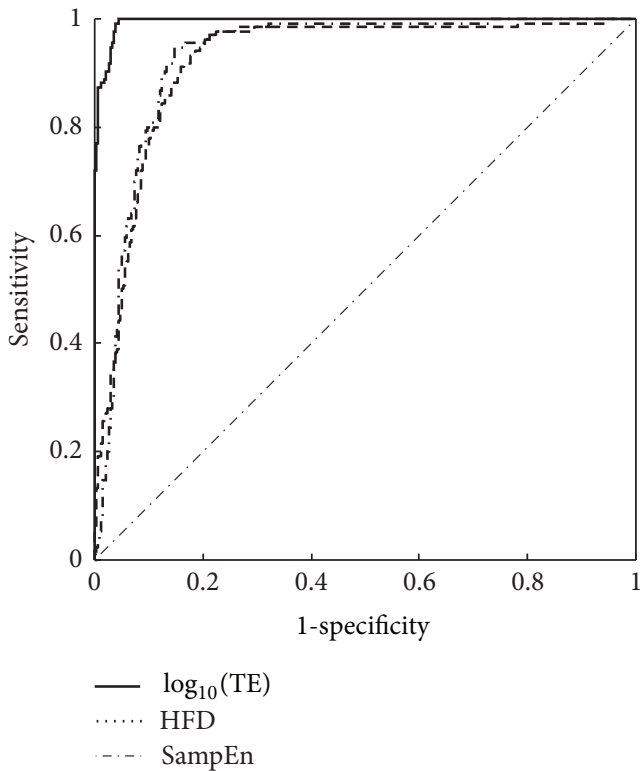

(d)

FIGURE 3: For classification problem-3 (a) the distribution of $\log _{10}$ (TE) values using box-whiskers plots (without outliers) for nonseizure and seizure groups. (b) The distribution of HFD values using box-whiskers plots (without outliers) for nonseizure and seizure groups. (c) The distribution of SampEn values using box-whiskers plots (without outliers) for nonseizure and seizure groups. (d) ROC curve for log l $_{10}$ (TE) (thick solid line), HFD (thick dotted line), and SampEn (thick dash-dot line) for discriminating nonseizure and seizure groups. The diagonal line (thin dash-dot line) from 0,0 to 1,1 represents ROC curve that cannot discriminate between nonseizure and seizure groups.

TABLE 4: Descriptive results of ROC analysis using TE, HFD, and SampEn for discriminating nonseizure and seizure groups (classification problem-3) for $W=3000$.

\begin{tabular}{lcccccc}
\hline Nonlinear method & AUC & Optimum threshold & Average sensitivity \% & Average specificity \% & Average precision \% & Average accuracy \% \\
\hline TE & 0.9960 & 3.5227 & 99.3 & 96.3 & 87.1 & 97.0 \\
HFD & 0.9246 & 1.0827 & 91.2 & 84.2 & 59.0 & 85.6 \\
SampEn & 0.9330 & 0.7962 & 91.9 & 86.8 & 63.5 & 87.8 \\
\hline
\end{tabular}

TABLE 5: Descriptive results of ROC analysis using TE, HFD, and SampEn for discriminating interictal and ictal groups of epileptic subjects (classification problem-4) for $W=3000$.

\begin{tabular}{lcccccc}
\hline Nonlinear method & AUC & Optimum threshold & Average sensitivity \% & Average specificity \% & Average precision \% & Average accuracy \% \\
\hline TE & 0.9978 & 3.5082 & 99.3 & 98.2 & 96.4 & 98.5 \\
HFD & 0.9533 & 1.0834 & 91.2 & 88.6 & 80.0 & 89.5 \\
SampEn & 0.8700 & 0.7761 & 89.7 & 75.4 & 64.6 & 80.1 \\
\hline
\end{tabular}




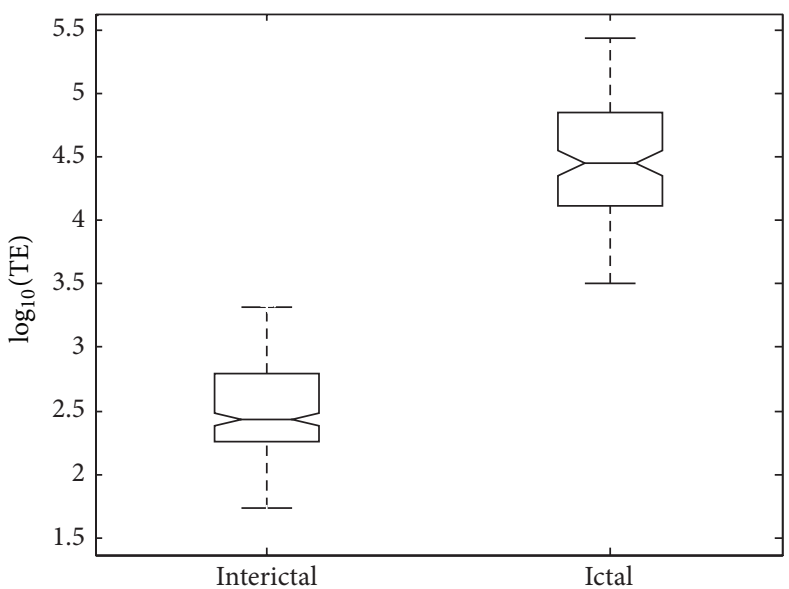

(a)

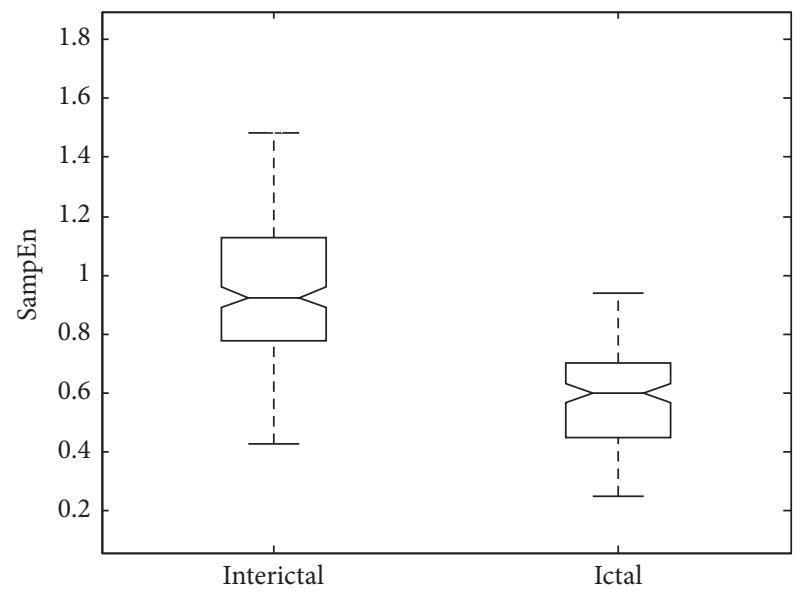

(c)

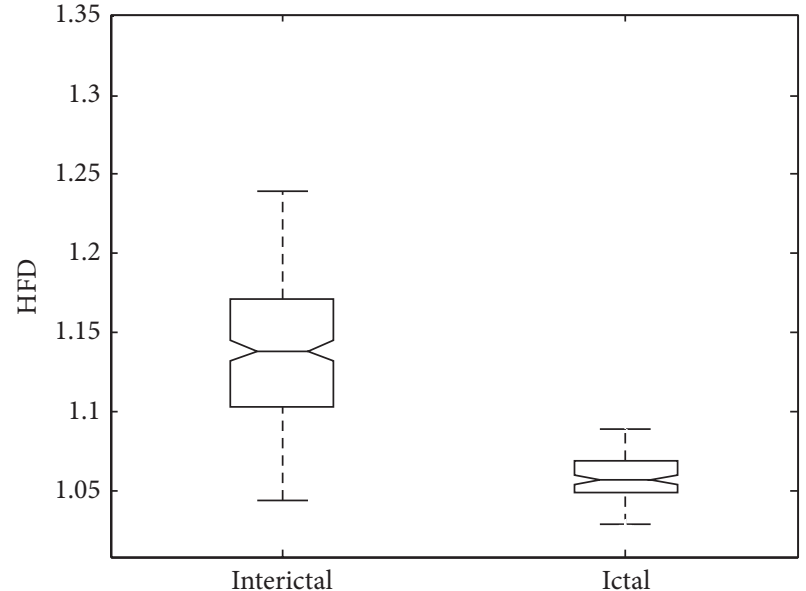

(b)

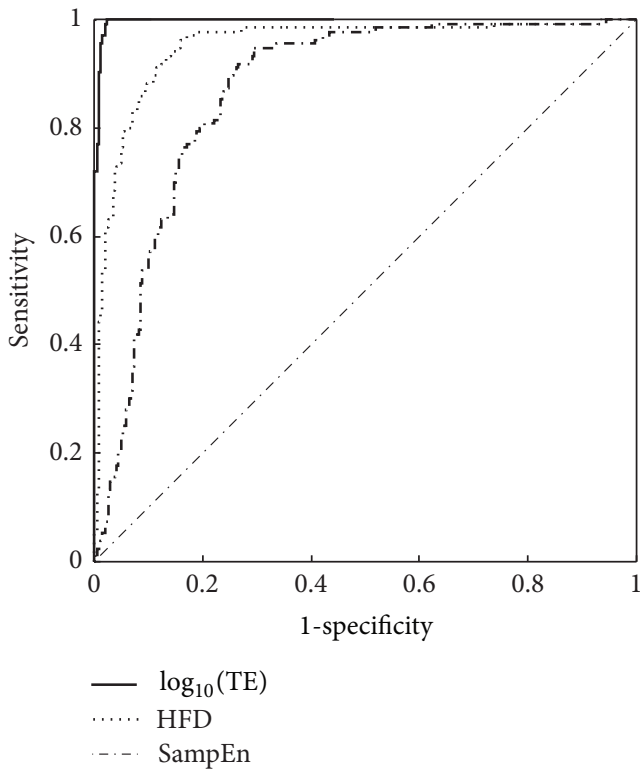

(d)

FIGURE 4: For classification problem-4 (a) the distribution of $\log _{10}$ (TE) values using box-whiskers plots (without outliers) for interictal and ictal groups. (b) The distribution of HFD values using box-whiskers plots (without outliers) for interictal and ictal groups. (c) The distribution of SampEn values using box-whiskers plots (without outliers) for interictal and ictal groups. (d) ROC curve for log 10 (TE) (thick solid line), HFD (thick dotted line), and SampEn (thick dash-dot line) for discriminating interictal and ictal groups. The diagonal line (thin dash-dot line) from 0,0 to 1,1 represents ROC curve that cannot discriminate between interictal and ictal groups.

measures this discrimination, that is, the ability of the test to correctly classify those with and without the disease and is regarded as an index of diagnostic accuracy. The optimum threshold is the cutoff point in which the highest accuracy (minimal false negative and false positive results) is obtained. This can be determined from the ROC curve as the closest value to the left top point (corresponding to $100 \%$ sensitivity and $100 \%$ specificity). An AUC value of 0.5 indicates that the test results are better than those obtained by chance, where as a value of 1.0 indicates a perfectly sensitive and specific test. A rough guide to categorize the precision of a diagnostic test based on AROC is as follows: the results are treated to be excellent if the AUC is between 0.9 and 1.0; if the AUC is between 0.8 and 0.89 , then the results are regarded to be good; the results are fair for values between 0.7 and 0.79 ; the results are poor for values between 0.6 and 0.69 ; if the AUC is between 0.5 and 0.59 , then the outcome indicates that the test results are not better than those obtained by chance.

\section{Results and Discussion}

As mentioned earlier, a 3000-sample sliding window with $90 \%$ overlap is used with every EEG segment from each dataset. We apply Kruskal-Wallis test to evaluate the statistical significance of TE, HFD, and SampEn for all the four classification problems. The results in terms of Chi-sq. and 


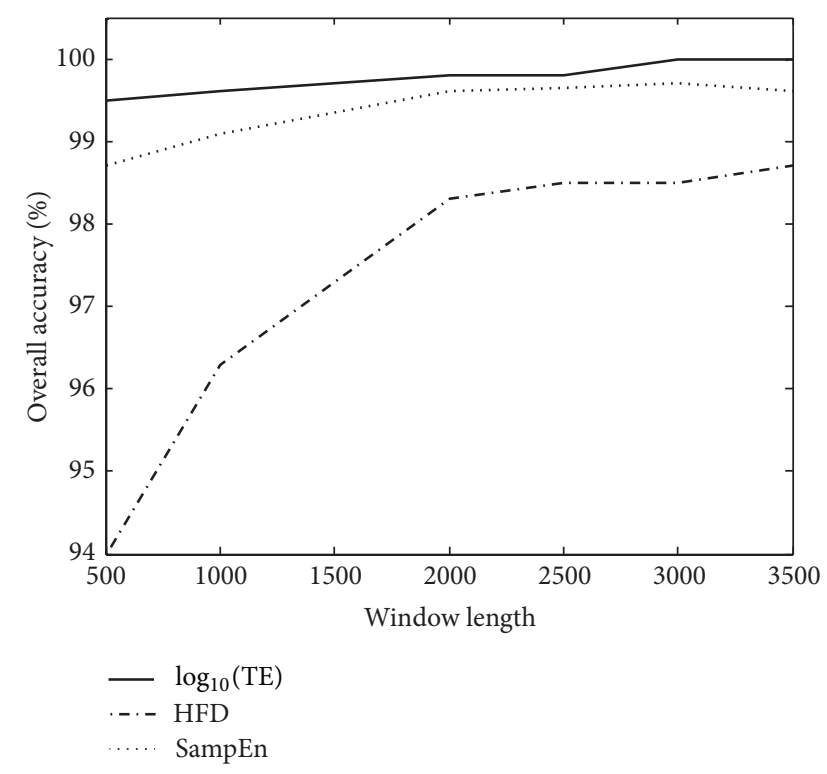

FIgURE 5: Plot of overall accuracy against window length, $W$, for TE, HFD, and SampEn analyses in separating normal from seizure group.

TABLE 6: Descriptive results of ROC analysis using TE for discriminating normal and seizure groups (classification problem-1) for different window lengths.

\begin{tabular}{lcccc}
\hline $\begin{array}{l}\text { Window } \\
\text { length, } \\
\begin{array}{l}W, \\
\text { samples }\end{array}\end{array}$ & $\begin{array}{c}\text { Window } \\
\text { length, } T, \\
\text { seconds }\end{array}$ & AUC & $\begin{array}{c}\text { Average } \\
\text { accuracy } \\
\%\end{array}$ & $\begin{array}{c}\text { Computation } \\
\text { time/window, } \\
\text { milliseconds }\end{array}$ \\
\hline 4000 & 23.04 & 1.0000 & 100.0 & 0.2519 \\
3000 & 17.28 & 1.0000 & 100.0 & 0.1990 \\
2000 & 11.52 & 0.9999 & 99.8 & 0.1657 \\
1000 & 5.76 & 0.9995 & 99.5 & 0.1283 \\
500 & 2.88 & 0.9988 & 99.4 & 0.1083 \\
200 & 1.15 & 0.9977 & 99.0 & 0.0996 \\
\hline
\end{tabular}

$P$ value are depicted in Table 1 . From the table it is found that all the three quantifiers are statistically significant and hence can be used to classify EEG signals. It is also found that among the three quantifiers TE performs the best. Since significant differences between classes are found, the ability of these nonlinear analyses methods to discriminate between the classes can be evaluated using receiver operating characteristic (ROC) plots in terms of AUC.

The distributions of TE, HFD, and SampEn for the normal and seizure classes in the classification problem-1 are shown using box-whiskers plots in Figures 1(a), 1(b), and 1(c), respectively. In Figure 1, the boxes (interquartile range) and the whiskers of normal and seizure groups are nonoverlapping for all the three nonlinear measures. This plot implies that all the three quantifiers can be readily used to distinguish between the normal and seizure groups of classification problem-1. Next to assess the diagnostic ability of each nonlinear method the ROC is plotted as shown in
Figure 1(d). Descriptive results of ROC analysis are tabulated in Table 2. From Table 2 it is found that the AUC is the highest for TE case, being equal to 1.0 with all the diagnostic parameters equal to $100 \%$. Between HFD and SampEn, the latter performs better with AUC $=0.9969$, sensitivity $=99.3 \%$, specificity $=100.0 \%$, positive predictivity $=100.0 \%$, and accuracy $=99.6 \%$. Since specificity for TE and SampEn cases is $100 \%$ each, it implies data is correctly classified and none is misclassified into another class. The classification accuracy for SampEn reaches 99.6\%; however, with TE it reaches $100 \%$, which implies TE is a better quantifier in separating normal from seizure group. The optimized threshold for the best separation is mentioned in Table 2. The distributions of TE, HFD, and SampEn for the nonseizure and seizure classes in the classification problem-2 are shown using boxwhiskers plots in Figures 2(a), 2(b), and 2(c), respectively. In Figure 2(a) the boxes (interquartile range) and the whiskers of nonseizure and seizure groups are nonoverlapping. In Figures 2(b) and 2(c) the boxes (interquartile range) of nonseizure and seizure groups are nonoverlapping while the whiskers are overlapping. Among the three quantifiers, obviously, TE shows the best discrimination. Now to assess the diagnostic ability of the three nonlinear methods the ROC is plotted as shown in Figure 2(d). The results of ROC analysis are tabulated in Table 3. The optimized threshold for best separation is also mentioned in the table. It is found that TE, HFD, and SampEn exhibit decreasing order of performance. This means that TE shows the best performance, while HFD performs better than SampEn for the classification problem-2. For classification problem-3, the distributions of TE, HFD, and SampEn for the nonseizure and seizure classes are shown using box-whiskers plots in Figures 3(a), 3(b), and 3(c), respectively. In Figure 3, the boxes (interquartile range) of nonseizure and seizure groups are nonoverlapping for all the three cases, while the whiskers are overlapping. The least overlap of whiskers is found in the case of TE. Again, to assess the diagnostic ability of the three nonlinear quantifiers the ROC is plotted as shown in Figure 3(d). Table 4 depicts the results of ROC analysis from which it is found that the AUC is the highest for TE case, being equal to 0.9960 with sensitivity $=99.3 \%$, specificity $=96.3 \%$, positive predictivity $=87.1 \%$, and accuracy $=97.0 \%$. Between HFD and SampEn, again the latter performs better with AUC $=0.9330$, sensitivity $=91.9 \%$, specificity $=86.8 \%$, positive predictivity $=63.5 \%$, and accuracy $=87.8 \%$. This implies that TE shows the best performance, while SampEn performs better than HFD for the classification problem3. The optimized threshold for the best separation is also specified in Table 4.

For classification problem-4, which is concerned with the separation of interictal and ictal classes of epileptic patients, the distributions of TE, HFD, and SampEn are shown using box-whiskers plots in Figures 4(a), 4(b), and 4(c), respectively. In Figure 4(a) the boxes (interquartile range) and the whiskers of interictal and ictal groups are nonoverlapping. However, in Figures 4(b) and 4(c) the boxes (interquartile range) of interictal and ictal groups are nonoverlapping while the whiskers are overlapping. Among the three quantifiers, again, TE shows best discrimination. 
TABLE 7: A comparison of classification accuracy achieved by our method and best performed others' method for three classification problems.

\begin{tabular}{|c|c|c|c|}
\hline Researcher (year) & Method & Dataset & $\begin{array}{l}\text { Classification } \\
\text { accuracy }(\%)\end{array}$ \\
\hline Tzallas et al. (2007) & Time-frequency analysis and ANN & $\mathrm{Z}, \mathrm{S}$ & 100.0 \\
\hline $\begin{array}{l}\text { Subasi and Gursoy } \\
(2010)\end{array}$ & $\begin{array}{l}\text { Principal component analysis, independent component analysis, linear } \\
\text { discriminant analysis, and support vector machines }\end{array}$ & $\mathrm{Z}, \mathrm{S}$ & 100.0 \\
\hline Guo et al. (2010) & Discrete wavelet transform, line length feature, and MLPNN & $\mathrm{Z}, \mathrm{S}$ & 99.6 \\
\hline Guo et al. (2011) & $\begin{array}{l}\text { Genetic programming based feature extraction and k-nearest-neighbors } \\
\text { classifier }\end{array}$ & $\mathrm{Z}, \mathrm{S}$ & 99.0 \\
\hline Wang et al. (2011) & Wavelet transform and Shannon entropy & $\mathrm{Z}, \mathrm{S}$ & 100.0 \\
\hline Iscan et al. (2011) & $\begin{array}{l}\text { Cross-correlation, power spectral density, support vector machines, Linear } \\
\text { discriminant analysis and k-nearest neighbors classifier }\end{array}$ & $\mathrm{Z}, \mathrm{S}$ & 100.0 \\
\hline Orhan et al. (2011) & Wavelet transform, k-nearest-neighbors classifier, and ANN & $\mathrm{Z}, \mathrm{S}$ & 100.0 \\
\hline This work (2013) & Teager energy feature and ROC & $\mathrm{Z}, \mathrm{S}$ & 100.0 \\
\hline Ocak (2009) & Discrete wavelet transform and approximate entropy & ZNF, S & 96.65 \\
\hline Guo et al. (2010) & Discrete wavelet transform, line length feature and MLPNN & ZNF, S & 97.75 \\
\hline This work (2013) & Teager energy feature and ROC & ZNF, S & 98.9 \\
\hline Tzallas et al. (2007) & Time-frequency analysis and ANN & ZONF, S & 97.73 \\
\hline Guo et al. (2010) & Discrete wavelet transform, line length feature, and MLPNN & ZONF, S & 97.77 \\
\hline Orhan et al. (2011) & Wavelet transform, k-nearest neighbors classifier and ANN & ZONF, S & 100.0 \\
\hline This work (2013) & Teager energy feature and ROC & ZONF, S & 97.0 \\
\hline This work (2013) & Teager energy feature and ROC & NF, S & 98.5 \\
\hline
\end{tabular}

To assess the performance ability of the three nonlinear quantifiers the ROC is plotted as shown in Figure 4(d). Table 5 shows the results of ROC analysis from which it is observed that the AUC is the highest for TE case, being equal to 0.9978 with sensitivity $=99.3 \%$, specificity $=98.2 \%$, precision $=96.4 \%$, and accuracy $=98.5 \%$. Between HFD and SampEn, however, the former performs better. These results substantiate our finding that TE can be preferred to HFD and SampEn in distinguishing nonseizure from seizure group. The optimized threshold for best separation in each case is also shown in Table 5. In practice, depending upon application, the threshold parameter can be optimized in a patient-specific way prioritizing higher sensitivity and lower false detection rate based on the ROC plot.

The rationale behind choosing 3000-sample analysis window for comparison is explained below. To arrive at near-optimal window length, first we study the impact of window length, $W$, on the TE, HFD, and SampEn. We compute these quantifiers for several window lengths of EEG from both nonseizure and seizure groups. For each window length we compute the overall accuracy in separating the two groups with each quantifier. Figure 5 shows the plot of overall accuracy against widow length, $W$, for the different quantifiers. It is found that as $W$ is increased from 500 to 3500 samples, the overall accuracy also increases and then almost saturates in all the three cases. But the effect is more predominant in the case of HFD (bottom curve shown by dash-dot line). Between TE and SampEn, TE is found to be largely independent of window length and showed relative consistency when used as a relative measure for comparison. From Figure 5 it is found that an optimum window length of
3000 samples (= 17.3 seconds) is well suited for comparison among the three quantifiers. It is also found that irrespective of window length TE performs the best.

Although in the above discussions we used a 3000-sample sliding window (with 90\% overlap between consecutive windows) with every EEG segment from each dataset, it is possible to use a shorter window for TE analysis, but at the cost of some overall accuracy. Table 6 shows the impact of window length, $W$, on the AUC and overall accuracy with TE analysis in discriminating normal and seizure classes. It is found that both AUC and overall accuracy decrease only marginally with a considerable decrease in window length. This implies that TE analysis can be carried out readily for shorter window lengths, too (see Figure 5). In other words, the mean TE quantifier can be used for short-term as well as long-term analysis. This may be attributed to the time resolution property of TEO which can capture rapid changes in the modulation energy. Since the best results (AUC = 1.0 and $100 \%$ accuracy) were shown for $W=3000$ and 4000 , we chose a moving window of length 3000 samples in the above discussion. Table 6 , also, depicts the computation time/window for different window lengths. As usual, a longer window length requires longer computation time.

We also ascertain the feasibility of executing the proposed TE method for real-time applications. All the three methods have been implemented using MATLAB programs. The average computational time for a window with 3000 samples, using TE method, is found to be 0.1990 milliseconds. This time interval is shorter compared to those for HFD (0.4141 milliseconds/window) and SampEn (1152.2 milliseconds/window) and hence, TE method is more suitable 
compared to HFD and SampEn, for automated offline/online EEG processing.

Various researchers have proposed different methods for epileptic seizure detection using the database by Andrzejak et al. [44]. Table 7 provides a comparison between our method and other methods that have used the same database. In the table, we present a listing of the method, dataset used, and classification accuracy, for the four classification problems. It should be noted that all the methods shown in the table, except ours, had used modern classifiers for first training and then classification. In the first classification problem, the results obtained by Tzallas, Subasi, and our method are the best (100\%). In the second problem, our method shows the best results (98.9\%). However, for the third classification problem, the result found by Orhan is the best (100\%). Our results, though not better, are nearly the same as most of others $(\approx 97 \%)$. For the fourth classification problem, we achieved an average accuracy of $98.5 \%$. Besides the feasibility of the proposed TE method for real-time implementations, the accuracy of diagnosis can be improved by increasing the number of features. An automated system developed based on this approach can provide feedback to the experts for quick and accurate EEG classification.

\section{Conclusion}

A novel method for EEG epileptic seizure detection based on TE is presented. The chief finding of this study is that the mean TE measure is largely independent of record length and shows relative consistency when used as a relative measure for comparison. We have also shown that the mean TE quantifier can be used for short-term as well as long-term analysis. The quantified TE is found to outperform the other two quantifiers (HFD and SampEn) in discriminating these EEG groups and thus can significantly add to the more efficient monitoring of epileptic patients. This nonlinear measure can easily be analyzed from ambulatory EEG recordings without time-consuming preprocessing and, hence may have practical implications for risk stratification too. The database used has already been preprocessed by the removal of artifacts by visual inspection. This is a limitation of our method (like many who have used the same database). Nevertheless, the results of this study provide sufficient evidence to warrant the assessment under actual clinical situations that can provide more robust confirmation of the application of TE as a reliable nonlinear quantifier to capture diagnostically significant information. Hence the method is well suited for real-time implementation in automated epilepsy monitoring system.

\section{Conflict of Interests}

There is no conflict of interests.

\section{Authors' Contributions}

The author, who is also the corresponding author, is the sole contributor to this work.

\section{References}

[1] L. D. Iasemidis, D.-S. Shiau, W. Chaovalitwongse et al., "Adaptive epileptic seizure prediction system," IEEE Transactions on Biomedical Engineering, vol. 50, no. 5, pp. 616-627, 2003.

[2] F. Lopes da Silva, W. Blanes, S. N. Kalitzin, J. Parra, P. Suffczynski, and D. N. Velis, "Epilepsies as dynamical diseases of brain systems: basic models of the transition between normal and epileptic activity," Epilepsia, vol. 44, no. 12, pp. 72-83, 2003.

[3] N. Mc Grogan, Neural network detection of epileptic seizures in the electroencephalogram [Ph.D. thesis], Oxford University, Oxford, UK, 1999.

[4] F. Mormann, R. G. Andrzejak, C. E. Elger, and K. Lehnertz, "Seizure prediction: the long and winding road," Brain, vol. 130, no. 2, pp. 314-333, 2007.

[5] J. Gotman, "Automatic detection of seizures and spikes," Journal of Clinical Neurophysiology, vol. 16, no. 2, pp. 130-140, 1999.

[6] J. Gotman, "Automatic recognition of epileptic seizures in the EEG," Electroencephalography and Clinical Neurophysiology, vol. 54, no. 5, pp. 530-540, 1982.

[7] A. M. Murro, D. W. King, J. R. Smith, B. B. Gallagher, H. F. Flanigin, and K. Meador, "Computerized seizure detection of complex partial seizures," Electroencephalography and Clinical Neurophysiology, vol. 79, no. 4, pp. 330-333, 1991.

[8] H. Qu and J. Gotham, "A patient-specific algorithm for the detection of seizure onset in long- term EEG monitoring: possible use as a warning device," IEEE Transactions on Biomedical Engineering, vol. 44, no. 2, pp. 115-122, 1997.

[9] N. Radhakrishnan and B. N. Gangadhar, "Estimating regularity in epileptic seizure time-series data: a complexity-measure approach," IEEE Engineering in Medicine and Biology Magazine, vol. 17, no. 3, pp. 89-94, 1998.

[10] J. Hu, J. Gao, and J. Principe, "Analysis of biomedical signals by the Lempel-Ziv complexity: the effect on finite data size," IEEE Transactions on Biomedical Engineering, vol. 53, no. 12, pp. 2606-2609, 2006.

[11] L. D. Iasemidis, H. P. Zaveri, J. C. Sackellares, W. J. Williams, and T. W. Hood, "Nonlinear dynamics of electrocorticographic data," Journal of Clinical Neurophysiology, vol. 5, p. 339, 1988.

[12] L. D. Iasemidis, J. C. Sackellares, H. P. Zaveri, and W. J. Williams, "Phase space topography and the lyapunov exponent of electrocorticograms in partial seizures," Brain Topography, vol. 2, no. 3, pp. 187-201, 1990.

[13] S. Gigola, F. Ortiz, C. E. D’Attellis, W. Silva, and S. Kochen, "Prediction of epileptic seizures using accumulated energy in a multiresolution framework," Journal of Neuroscience Methods, vol. 138, no. 1-2, pp. 107-111, 2004.

[14] O. A. Rosso, S. Blanco, J. Yordanova et al., "Wavelet entropy: a new tool for analysis of short duration brain electrical signals," Journal of Neuroscience Methods, vol. 105, no. 1, pp. 65-75, 2001.

[15] X. Li, G. Ouyang, and D. A. Richards, "Predictability analysis of absence seizures with permutation entropy," Epilepsy Research, vol. 77, no. 1, pp. 70-74, 2007.

[16] O. A. Rosso, "Entropy changes in brain function," International Journal of Psychophysiology, vol. 64, no. 1, pp. 75-80, 2007.

[17] V. Srinivasan, C. Eswaran, and N. Sriraam, "Approximate entropy-based epileptic EEG detection using artificial neural networks," IEEE Transactions on Information Technology in Biomedicine, vol. 11, no. 3, pp. 288-295, 2007.

[18] J. Gao, C. Yinhe, W. Ten, and J. Hu, Multiscale Analysis of Complex Time Series: Integration of Chaos and Random Fractal 
Theory, and Beyond, John Wiley \& Sons, Hoboken, NJ, USA, 2007.

[19] J. Gao, J. Hu, and W. Tung, "Complexity measures of brain wave dynamics," Cognitive Neurodynamics, vol. 5, pp. 171-182, 2011.

[20] E. I. Plotkin and M. N. S. Swamy, "Multistage implementation of parameterinvariant null filter and its application to discrimination of closely spaced sinusoids," in Proceedings of the IEEE International Symposium on Circuits and Systems, vol. 1, pp. 767770, 1988.

[21] E. I. Plotkin and M. N. S. Swamy, "Parameter-free structural modeling: a contribution to the solution of the separation of highly correlated AR-signals," in Proceedings of the IEEE International Symposium on Circuits and Systems (ISCAS '98), pp. 1-4, June 1998.

[22] E. I. Plotkin and M. N. S. Swamy, "Signal processing based on parameter structural modeling and separation of highly correlated signals of known structure," Circuits, Systems, and Signal Processing, vol. 17, no. 1, pp. 51-68, 1998.

[23] B. Litt, R. Esteller, J. Echauz et al., "Epileptic seizures may begin hours in advance of clinical onset: a report of five patients," Neuron, vol. 30, no. 1, pp. 51-64, 2001.

[24] T. F. Quatieri, Discrete-Time Speech Signal Processing, Principles and Practice, Pearson Education Pte., Singapore, 2004.

[25] J. F. Kaiser, "On a simple algorithm to calculate the "energy" of a signal," in Proceedings of the International Conference on Acoustics, Speech, and Signal Processing, pp. 381-384, April 1990.

[26] T. L. Nwe, S. W. Foo, and L. C. De Silva, "Classification of stress in speech using linear and nonlinear features," in Proceedings of the IEEE International Conference on Accoustics, Speech, and Signal Processing, pp. 9-12, April 2003.

[27] L. Atlas and J. Fang, "Quadratic detectors for general nonlinear analysis of speech," Proceedings of the IEEE International Conference on Acoustics, Speech and Signal Processing, vol. 2, pp. 9-12, 1992.

[28] D. A. Cairns and J. H. L. Hansen, "Nonlinear analysis and classification of speech under stressed conditions," Journal of the Acoustical Society of America, vol. 96, no. 6, pp. 3392-3400, 1994.

[29] C. Kamath, "A new approach to detect congestive heart failure using Teager energy nonlinear scatter plot of R-R interval series," Medical Engineering and Physics, vol. 34, no. 7, pp. 841848, 2012.

[30] C. Kamath, "ECG beat classification using features extracted from Teager energy functions in time and frequency domains," IET Signal Processing, vol. 5, no. 6, pp. 575-581, 2011.

[31] H. P. Zaveri, W. J. Williams, and J. C. Sackellares, "Energy based detection of seizures," in Proceedings of the 15th Annual International Conference of the IEEE Engineering in Medicine and Biology Society, pp. 363-364, October 1993.

[32] R. Esteller, Detection of seizure onset in epileptic patients from intracranial EEG signals [Ph.D. thesis], Georgia Institute of Technology, Department of Electrical and Computer Engineering, Atlanta, Ga, USA, 2000.

[33] A. B. Gardner, A. M. Krieger, G. Vachtsevanos, and B. Litt, "One-class novelty detection for seizure analysis from intracranial EEG," Journal of Machine Learning Research, vol. 7, pp. 1025-1044, 2006.

[34] R. Agarwal and J. Gotman, "Adaptive segmentation of electroencephalographic data using a nonlinear energy operator," in Proceedings of the IEEE International Symposium on Circuits and Systems (ISCAS '99), pp. V-199-V-202, June 1999.
[35] M. D’Alessandro, R. Esteller, G. Vachtsevanos, A. Hinson, J. Echauz, and B. Litt, "Epileptic seizure prediction using hybrid feature selection over multiple intracranial EEG electrode contacts: a report of four patients," IEEE Transactions on Biomedical Engineering, vol. 50, no. 5, pp. 603-615, 2003.

[36] R. Yadav, R. Agarwal, and M. N. S. Swamy, "Detection of epileptic seizures in stereo-EEG using frequency-weighted energy," in Proceedings of the 50th Midwest Symposium on Circuits and Systems (MWSCAS '07), pp. 77-80, August 2007.

[37] W. Klonowski, "Application of new non-linear dynamics methods in biosignal analysis," in World Medical Conference, vol. 5975 of Proceedings of SPIE, pp. 335-344, 2006.

[38] W. Klonowski, "From conformons to human brains: an informal overview of nonlinear dynamics and its applications in biomedicine," Nonlinear Biomedical Physics, vol. 1, article 5, 2007.

[39] W. Klonowski, "Personalized neurological diagnostics from biomedical Physicist's point of view and application of new nonlinear dynamics methods in biosignal analysis," International Journal of Biology and Biomedical Engineering, vol. 5, no. 4, pp. 190-200, 2011.

[40] T. Q. D. Khoa, V. Q. Ha, and V. V. Toi, "Higuchi fractal properties of onset epilepsy electroencephalogram," Computational and Mathematical Methods in Medicine, vol. 2012, Article ID 461426, 6 pages, 2012.

[41] S. M. Pincus and A. L. Goldberger, "Physiological time-series analysis: what does regularity quantify?" American Journal of Physiology, vol. 266, no. 4, pp. H1643-H1656, 1994.

[42] J. S. Richman and J. R. Moorman, "Physiological time-series analysis using approximate and sample entropy," American Journal of Physiology, vol. 278, no. 6, pp. H2039-H2049, 2000.

[43] S. Ramdani, B. Seigle, J. Lagarde, F. Bouchara, and P. L. Bernard, "On the use of sample entropy to analyze human postural sway data," Medical Engineering and Physics, vol. 31, no. 8, pp. 10231031, 2009.

[44] R. G. Andrzejak, K. Lehnertz, F. Mormann, C. Rieke, P. David, and C. E. Elger, "Indications of nonlinear deterministic and finite-dimensional structures in time series of brain electrical activity: dependence on recording region and brain state," Physical Review E, vol. 64, no. 6, Article ID 061907, 8 pages, 2001.

[45] L. Guo, D. Rivero, J. Dorado, J. R. Rabuñal, and A. Pazos, "Automatic epileptic seizure detection in EEGs based on line length feature and artificial neural networks," Journal of Neuroscience Methods, vol. 191, no. 1, pp. 101-109, 2010.

[46] F. Amor, S. Baillet, V. Navarro, C. Adam, J. Martinerie, and M. Le Van Quyen, "Cortical local and long-range synchronization interplay in human absence seizure initiation," NeuroImage, vol. 45, no. 3, pp. 950-962, 2009.

[47] A. Aarabi, F. Wallois, and R. Grebe, "Does spatiotemporal synchronization of EEG change prior to absence seizures?" Brain Research, vol. 1188, no. 1, pp. 207-221, 2008.

[48] W. Klonowski, "Everything you wanted to ask about EEG but were afraid to get the right answer," Nonlinear Biomedical Physics, vol. 3, article 2, 2009.

[49] T. Higuchi, "Approach to an irregular time series on the basis of the fractal theory," Physica D, vol. 31, no. 2, pp. 277-283, 1988.

[50] H. Hinrikus, M. Bachmann, D. Karai et al., "Higuchi's fractal dimension for analysis of the effect of external periodic stressor on electrical oscillations in the brain," Medical and Biological Engineering and Computing, vol. 49, no. 5, pp. 585-591, 2011. 
[51] M. H. Zweig and G. Campbell, "Receiver-operating characteristic (ROC) plots: a fundamental evaluation tool in clinical medicine," Clinical Chemistry, vol. 39, no. 4, pp. 561-577, 1993. 

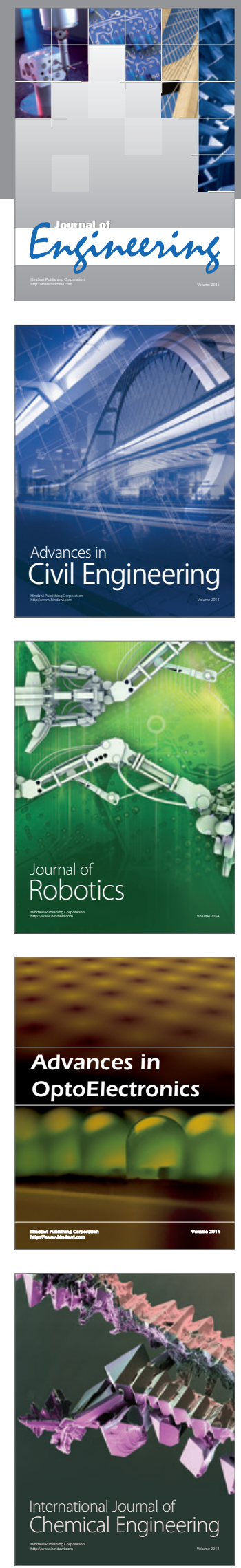

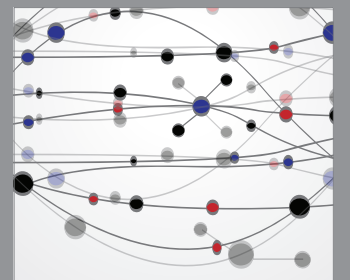

The Scientific World Journal
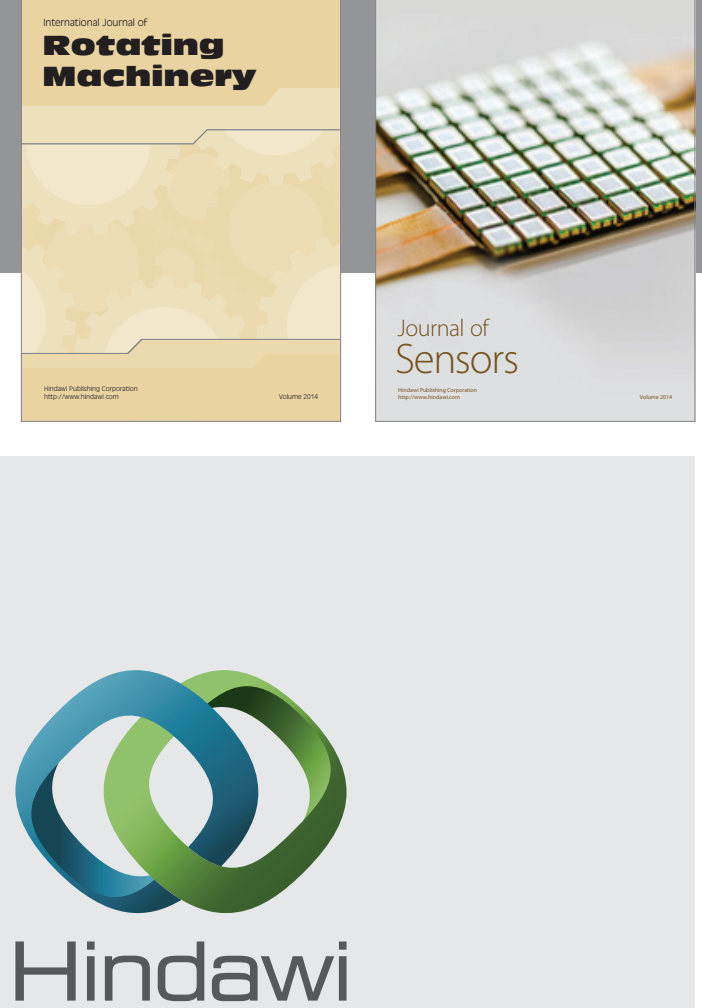

Submit your manuscripts at http://www.hindawi.com
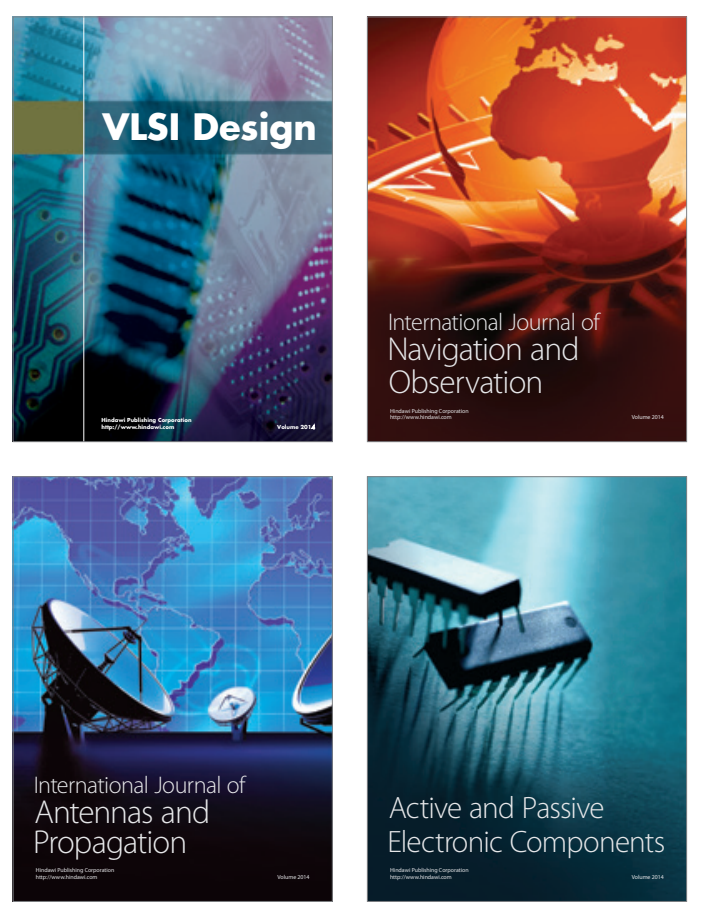
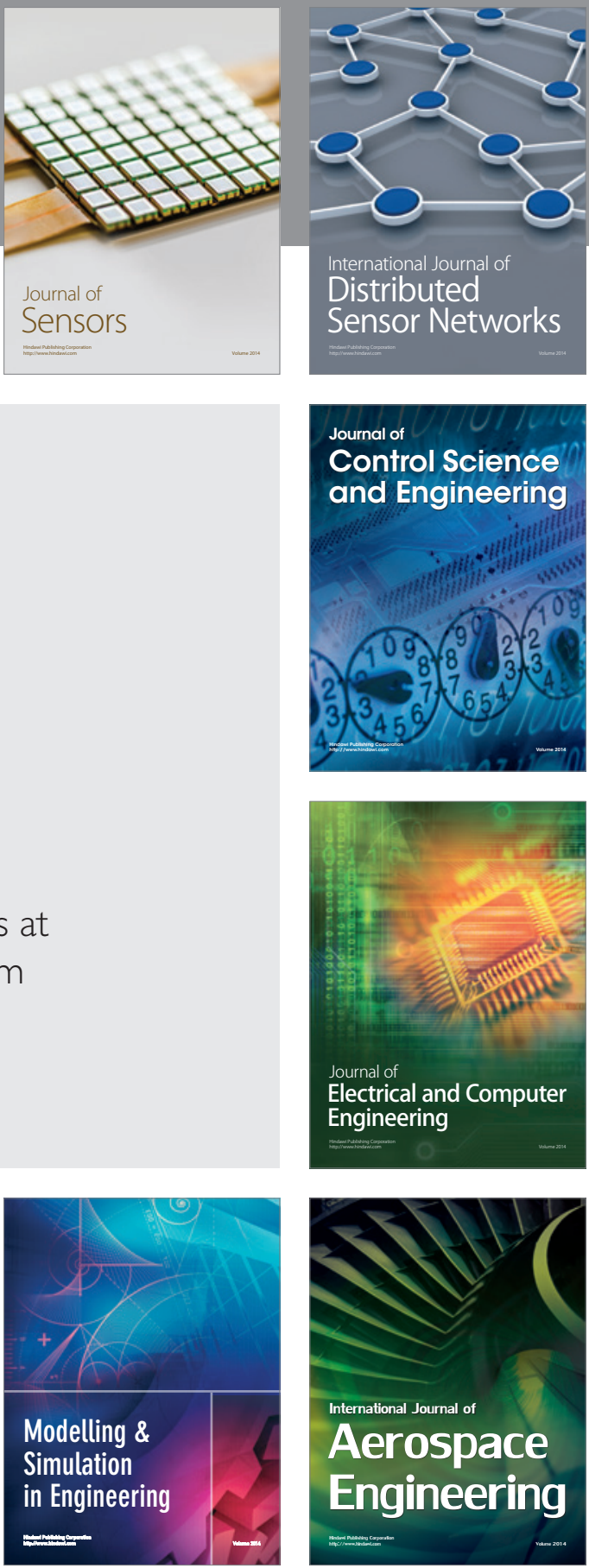

Journal of

Control Science

and Engineering
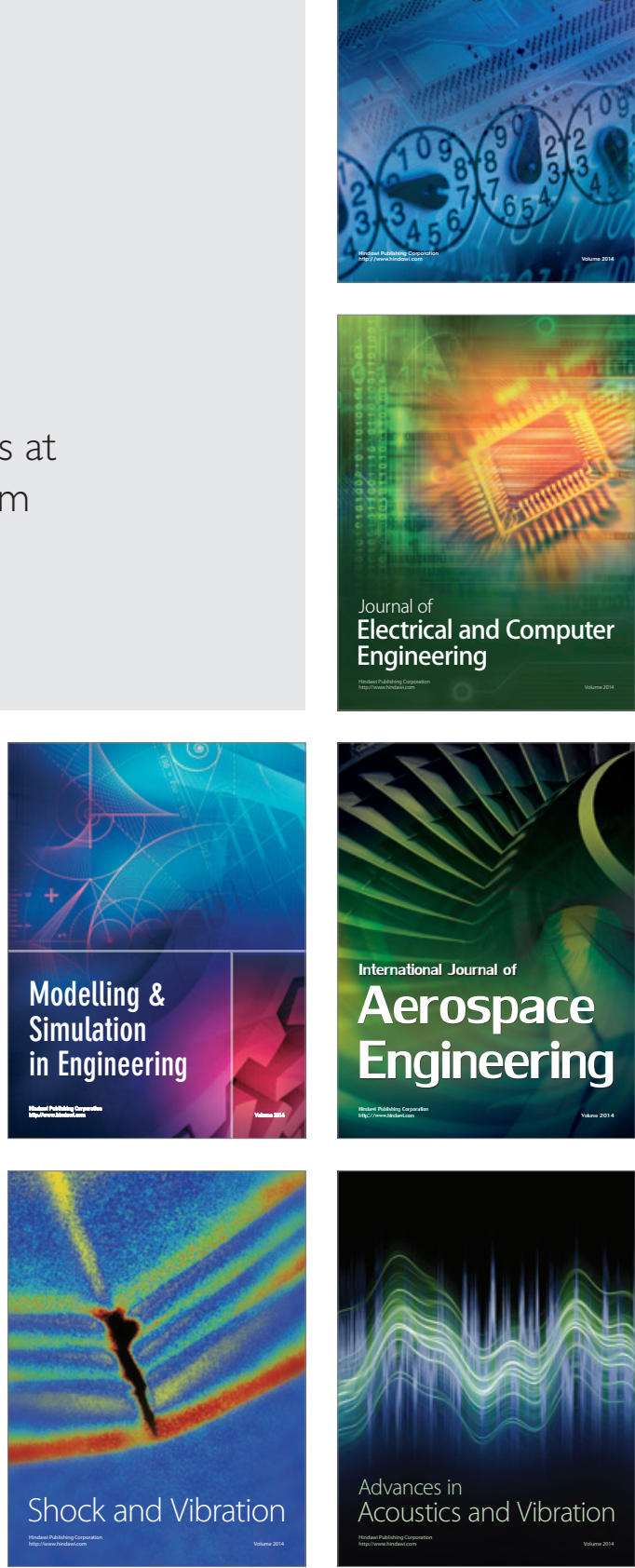